\title{
ON THE DIFFERENTIAL GEOMETRY OF TANGENT BUNDLES OF RIEMANNIAN MANIFOLDS
}

\author{
SHIGEO SASAKI
}

(Receive1 September 5, 1958)

Introduction. Let $M^{n}$ be an $n$-dimensional differentiable manifold. The set of all tangent vectors of $M^{n}$ constitutes, with a natural topology, the so-called tangent bundle of $M^{n}$. We denote it by $T\left(M^{n}\right)$. The set of all unit vectors of $M^{n}$ constitutes a subbundle of $T\left(M^{n}\right)$ and is a sphere-bundle over $M^{n}$. It is called the tangent sphere-bundle of $M^{n}$. We shall denote it by $T_{1}\left(M^{n}\right)$.

H. Poincaré used the tangent sphere-bundles of ovaloids in three dimensional Euclidean space, i.e. the phase spaces of the ovaloids, to prove the existence of certain closed geodesics on the ovaloids. He introduced a Riemannian metric on the tangent sphere-bundles and considered the geodesic flow on it. As the metric of tangent bundles of Riemannian manifolds seems to be important, we would like to study differential geometry of tangent bundles of Riemannian manifolds by introducing on it natural Riemannian metrics. In this papar we shall do it by restricting ourselves only to the tangent bundles $T\left(M^{n}\right)$.

1. Incompressible vector fields over a Riemannian manifold $\boldsymbol{M}^{n}$. Let $\xi$ be a differentiable vector field over a differentiable Riemannian manifold $M^{n}$ and suppose its components with respect to an arbitrary coordinate neighborhood $U$ be $\xi^{i}{ }^{1)}$ The following Lemma is well-known:

LEMMA 1. In order that the infinitesimal transformation $X f=\xi^{i} \frac{\partial f}{\partial x^{i}}$ leaves invariant volume element of the Riemannian manifold $M^{n}$, it is necessary and sufficient that the divergence

$$
\xi_{, i}^{i}=0
$$

identically, where the comma in $\xi_{, i}^{i}$ means the covariant derivative.

If the divergence of a vector field $\xi$ over $M^{n}$ vanishes identically, it is clear that the one parameter group of transformations of $M^{\prime 2}$ generated by $X f=\xi^{i} \frac{\partial f}{\partial x^{i}}$ is a group whose elements are homeomorphisms which leave invariant volumes of all domains in $M^{n}$. On account of this fact we shall say that $\xi$ is an incompressible vector field if the divergence of the vector field

1) We assume that groups of indices located in the left hand side of the following lines take values which lie on the right hand side of the corresponding lines.

$$
\begin{aligned}
& h, i, j, k, l ; a, b, c \\
& \lambda . \mu . \nu, \rho, \sigma ; \alpha, \beta, \gamma=1,2, \ldots \ldots \ldots \ldots n, \\
& H, I, J, K ; A, B, C=1.2, \ldots \ldots \ldots .2 n .
\end{aligned}
$$


vanishes identically.

ExAmple 1. A Killing vector field $\xi$ of $M^{n}$ is characterized by the differential equation

$$
\xi_{i, j}+\xi_{j, i}=0
$$

where $\xi_{i}=g_{i j} \xi^{j}, g_{i j}$ being components of the fundamental tensor of $M^{n}$. So, $\xi^{i}$ satisfies

$$
\xi_{, i}=0 . \quad .
$$

Hence, every Killing vector field of $M^{n}$ is an incompressible vector field. This is geometrically evident, because the infinitesimal transformation $\boldsymbol{X} f=\xi^{i} \frac{\partial f}{\partial x^{i}}$, where $\xi^{i}$ are components of a Killing vector field, generates a one-parameter group of isomeiries. It is well known that (1.2) is also written as

$$
\frac{\partial g_{j k}}{\partial x^{i}} \xi^{i}+\frac{\partial \xi^{i}}{\partial x^{k}} g_{j i}+\frac{\partial \xi^{i}}{\partial x^{j}} g_{i k}=0 .
$$

EXAmple 2. A harmonic vector field $\xi$ is characterized by the differential equations

$$
\xi_{i, j}=\xi_{j, i}, \quad \xi_{, i}^{i}=0,
$$

where $\xi_{i}=g_{i j} \xi^{j}$. Hence, every harmonic vector field of $M^{n}$ is also an incompressible vector field.

Lemma 2. Let $\xi$ and $\eta$ be two incompressible vector fields in a Riemannian manifold $M^{n}$, then their Poisson bracket $[\xi, \eta]$ is also an incompressible vector field.

Proof. By definition the $i$-components of $[\xi, \eta]$ is given by

Hence, we get

$$
\begin{aligned}
{[\xi, \eta]^{i} } & =\xi^{j} \frac{\partial \eta^{i}}{\partial x^{j}}-\eta^{j} \frac{\partial \xi^{i}}{\partial x^{j}} \\
& =\xi^{j} \eta_{, g}^{i}-\eta^{j} \xi_{, j}^{i} .
\end{aligned}
$$

$$
[\xi, \eta]_{, i}^{i}=\xi^{j} \eta_{, j i}^{i}-\eta^{j} \xi_{, j i}^{i} .
$$

However, if we use the well-known relation

$$
\xi_{, j k}^{i}-\xi_{, k j}^{i}=R_{h j k}^{i} \xi^{h}
$$

where $R_{h y k}^{\prime}$ 's are the components of the curvature tensor, we can easily see that

$$
\begin{aligned}
\xi_{, j i}^{i} & =\xi_{, i j}^{i}+R_{k j j}^{i} \xi^{k} \\
& =R_{k j} \xi^{k},
\end{aligned}
$$

$R_{k j}$ being the components of the Ricci tensor. Accordingly we see

$$
[\xi, \eta]_{, i}^{i}=\xi^{j} \eta^{k} R_{k j}-\eta^{j} \xi^{k} R_{k j}=0,
$$

which is to be proved. 
In spite of Lemma 2 it seams to us that all incompressible vector fields of a Riemannian manifold $M^{3}$ do not ganerate, in general, a finite continuous group of homeomorphisms which leave invariant volume of $M^{n}$, but do generate an infinite continuous group.

2. Extended transformations and extended tensors. Let $T\left(M^{n}\right)$ be the set of all tangent vectors of $M^{n}$. The $T\left(M^{n}\right)$, with the natural topology, has a bundle structure with $M^{n}$ as the base space, linear vector space $E^{n}$ as the standard fibre and the group $G L(n)$ as the structural group. We call this bundle the tangent bunlle of $M^{n}$ and denote the natural projection $T\left(M^{n}\right) \rightarrow M^{n}$ by $\pi$. The bundle $T\left(M^{n}\right)$ has a cross section corresponding to zero vectors. We may identify $M^{n}$ with this cross section.

Now, let $U$ be a coordinate neighborhood of $M^{n}$ with $n$ variables $x^{i}$ as coordinates. Then every tangent vector of $M^{n}$ at $P \in U$, can be expressed by $2 n$ variables $\left(x^{i}, v^{i}\right)$ where $x^{i}$ are coordinates of the point $P$ with respect to the coordinate neighborhood and $v^{i}$ are components of the tangent vector in consideration with respect to the natural frame of reference at $P$, i. e. the frame constituted by the vectors $\frac{\partial}{\partial x^{i}}$ at $P$. So, we may consider $U \times E^{n}$ as a coordinate neighborhood of $T\left(M^{n}\right)$.

Let $U\left(x^{i}\right), U^{\prime}\left(x^{\prime a}\right)$ be two coordinate neighborhoods of $M^{n}$ such that $U \cap U^{\prime}$ is not empty. Then, the intersection of two coordinate neighborhoods $U \times E^{n}\left(x^{i}, v^{i}\right)$ and $U^{\prime} \times E^{n}\left(x^{\prime a}, v^{\prime a}\right)$ of $T\left(M^{n}\right)$ is not empty too. To the coordinate transformation

$$
x^{\prime a}=x^{\prime a}\left(x^{1}, \ldots, x^{n}\right)
$$

of $U \cap U^{\prime}$, there corresponds the coordinate transformation

$$
\left\{\begin{array}{l}
x^{a}=x^{\prime a}\left(x^{1}, \ldots, x^{n}\right) \\
\boldsymbol{v}^{\prime a}=\frac{\partial x^{\prime a}}{\partial x^{i}} v^{i}
\end{array}\right.
$$

of $\left(U \times E^{n}\right) \cap\left(U^{\prime} \times E^{n}\right)$. We shall say that (2.2) is the extended transfor mation of (2.1).

For every coordinate transformation of $M^{n}$, there corresponds a matrix $\left(\frac{\partial x^{\prime a}}{\partial x^{i}}\right)$. Making use of this matrix, the transposed matrix $\left(\frac{\partial x^{i}}{\partial x^{\prime a}}\right)$ and their Kronecker products, we used to define tensors of $M^{n}$. If we put

$$
x^{n+i} \equiv v^{i}, x^{n+a} \equiv v^{\prime a} \text {, }
$$

for the sake of convenience, we may write (2.2) as

$$
x^{\prime A}=x^{\prime A}\left(x^{1}, \ldots, x^{n}, x^{n+1}, \ldots, x^{2 n}\right) \text {. }
$$

So we may define tensors of $T\left(M^{n}\right)$ by making use of a matrix $\left(\frac{\partial x^{\prime A}}{\partial x^{I}}\right)$ i.e.

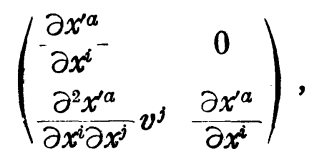

its transposed matrix and their Kronecker products. They are special cases 
of extensors developed extensively by H. V.Craig and A. Kawaguchi.

Let $\xi$ be a contravariant (covariant) vector field on $M^{n}$ and $\xi^{i}\left(\xi_{i}\right)$ be its components with respect to a coordinate neighborhood $U\left(x^{i}\right)$. If we put

$$
\begin{aligned}
& \bar{\xi}^{i} \equiv \xi^{i}, \bar{\xi}^{n+i} \equiv \frac{\partial \xi^{i}}{\partial x^{j}} v^{j}, \\
& \bar{\xi}_{i} \equiv \frac{\partial \xi_{i}}{\partial x^{j}} v^{j}, \bar{\xi}_{n+i} \equiv \xi_{i},
\end{aligned}
$$

then we may easily verify that $\bar{\xi}^{I}\left(\bar{\xi}_{I}\right)$ are components of a contravariant (covariant) vector field of $T\left(M^{n}\right)$ with respect to the coordinate neighborhood $U \times E^{n}\left(x^{i}, v^{i}\right)$. We shall call the vector field $\xi$ of $T\left(M^{n}\right)$ thus derived from the one of $M^{n}$ an extended vector field or an extension of a contravariant (covariant) vector field $\xi$ of $M^{n}$.

In the same way, if $\xi^{i j}\left(\xi_{j}^{i}\right.$ and $\left.\xi_{i j}\right)$ 's are components of a contravariant (mixed and covariant) tensor field of $M^{n}$ with respect to $U\left(x^{i}\right)$, then $\bar{\xi}^{I J}\left(, \xi_{J}^{r}\right.$ and $\bar{\xi}_{I J}$ 's defined by

$$
\begin{aligned}
& \bar{\xi}^{i j}=0, \bar{\xi}^{i n+j}=\bar{\xi}^{n+i j}=\xi^{i j}, \quad \bar{\xi}^{n+i n+j}=\frac{\partial \xi^{i j}}{\partial x^{k}} v^{k}, \\
& \bar{\xi}_{j}^{l}=\xi_{j}^{i}, \bar{\xi}_{n+j}^{\prime}=0, \bar{\xi}_{j}^{n+i}=\frac{\partial \xi_{j}^{i}}{\partial x^{k}} v^{k}, \bar{\xi}_{n+j}^{n+i}=\xi_{j}^{i}, \\
& \bar{\xi}_{i j}=\frac{\partial \xi_{i j}}{\partial x^{k}} v^{k}, \bar{\xi}_{i n+j}=\bar{\xi}_{n+i j}=\xi_{i j,}, \bar{\zeta}_{n+i n+j}=0,
\end{aligned}
$$

are components of a contravariant (mixed and covariant) tensor field of $T\left(M^{n}\right)$ with repsect to $U \times E^{n}\left(x^{i}, v^{i}\right)$. We shall call this $\bar{\xi}$ the extended tensor field or an extension of the contravariant (mixed and covariant) tensor field $\xi$ of $M^{n}$.

When $\xi^{i}$ 's are components of a contravariant vector field of $M^{n}$, then $\left(0, \xi^{i}\right)$ are components of a contravariant vector field of $T\left(M^{n}\right)$. Hence, it follows that $\left(0, \xi_{, j}^{i} v^{j}\right)$ are components of a contravariant vector field of $T\left(M^{n}\right)$. If we subtract the last vector field from the extension of $\xi$, we can see that the following Lemma is true.

LEMMA 3. If $\xi^{i}$ 's are components of a contravariant vector field of $M^{n}$, then $\left(0, \xi^{i}\right)$ and $\left(\xi^{i},-\left\{\begin{array}{c}i \\ j k\end{array}\right\} \xi^{j} v^{k}\right)$ are contravariant vector fields of $T\left(M^{n}\right)$.

COROLlARY. Suppose that $U$ be a coordinate neighborhood of $M^{n}$ and take a contravariant vector field of $T\left(M^{n}\right)$ whose components with respect to $U \times E^{n}$ are $\left(v^{i},-\left\{\begin{array}{c}i \\ j k\end{array}\right\} v^{j} v^{k}\right)$. Then, the vector field in $U \times E^{n}$ has the same form in $\left(U \times E^{n}\right) \cap\left(V \times E^{n}\right)$ for every coordinate neighborhood $V \times E^{n}$ such that $U \cap V$ is not empty.

In the same way we can state the following Lemma.

LEMMA 4. If $\xi_{i}$ 's are components of a covariant vector field of $M^{n}$, then 
$\left(\xi_{i}, 0\right)$ and $\left(\left\{\begin{array}{c}h \\ i j\end{array}\right\} \xi_{h} v^{j}, \xi_{i}\right)$ are covariant vector fields of $T\left(M^{n}\right)$.

3. The Riemannian metric of $\boldsymbol{T}\left(\boldsymbol{M}^{n}\right)$. Let $g_{j k}$ be the fundamental metric tensor of $M^{n}$ with respect to a coordinate neighborhood $U\left(x^{i}\right)$. Then

$$
d s^{2}=g_{j k}(x) d x^{j} d x^{k}
$$

is the line element of the Riemannian manifold $M^{n}$. Now we shall define a line element of $T\left(M^{n}\right)$ in $U \times E^{n}$ by

$$
d \sigma^{2}=g_{j k}(x) d x^{j} d x^{k}+g_{j k}(x) D v^{j} D v^{k}
$$

where $D v^{j}$ means the covariant differential of $v^{j}$, i.e.

$$
D v^{j}=d v^{j}+\left\{\begin{array}{c}
j \\
l m
\end{array}\right\} v^{l} d x^{m},
$$

$\left\{\begin{array}{c}j \\ l m\end{array}\right\}$ being Christoffel's symbols. It is clear that the right hand side of (3.3) is invariant under coordinate transformations of $M^{n}$ and positive definite. In the following we shall consider the tangent bundle $T\left(M^{n}\right)$ as a Reimannian manifold endowed with the metric (3.2).

Now, the components of the fundamental (covariant) metric tensor of $T\left(M^{n}\right)$ with respect to $U \times E^{n}\left(x^{i}, v^{i}\right)$ can be obtained by putting (3.2) in the form

$$
d \sigma^{2}=G_{J K} d x^{J} d x^{K}
$$

We can easily see that

$$
\left\{\begin{array}{l}
G_{j k}=g_{j k}+g_{\beta \gamma}\left\{\begin{array}{c}
\beta \\
\mu j
\end{array}\right\}\left\{\begin{array}{c}
\gamma \\
\nu k
\end{array}\right\} v^{\mu} v^{\nu}, \\
G_{j n+k}=[\lambda j, k] v^{\lambda}, \\
G_{n+j n+k}=g_{j k},
\end{array}\right.
$$

where $[\lambda j, k],\left\{\begin{array}{c}\beta \\ \mu k\end{array}\right\}$ are Christoffel's symbols of the first and second kind of $M^{n}$ respectively.

We can explicitly give the contravariant components of the fundamental tensor of $T\left(M^{n}\right)$. This is easily done by solving the equation

$$
G_{J K} G^{I K}=\delta_{J}^{I}
$$

thinking $G^{I K}$ as unknown variables. The result is as follows:

$$
\left\{\begin{array}{l}
G^{j k}=g^{j k}, \\
G^{j n+k}=-\left\{\begin{array}{c}
k \\
\mu !
\end{array}\right\} g^{j l} v^{\mu}, \\
G^{n+j n+k}=g^{j k}+g^{g \gamma}\left\{\begin{array}{c}
i \\
\mu \beta
\end{array}\right\}\left\{\begin{array}{c}
k \\
\nu \gamma
\end{array}\right\} v^{\mu} v^{\nu} .
\end{array}\right.
$$

The condition of orthogonality

$$
G_{B C} d x^{B} \delta x^{C}=0
$$

of two vectors $d x^{4}, \delta x^{4}$ of $T\left(M^{n}\right)$ can be written also as

$$
g_{j k} d x^{j} \delta x^{k}+g_{j k} D v^{j} \Delta v^{k}=0
$$

provided that 


$$
\begin{aligned}
& D v^{j}=d v^{j}+\left\{\begin{array}{c}
j \\
l m
\end{array}\right\} v^{l} d x^{m}, \\
& \Delta v^{j}=\delta v^{j}+\left\{\begin{array}{c}
j \\
l m
\end{array}\right\} v^{l} \delta x^{m} .
\end{aligned}
$$

Tangent vectors of the fibre at a point $P\left(x^{i}, v^{i}\right) \in U \times E^{n}$ have components of the form $\left(0, \delta v^{i}\right)$. Hence, we can easily see that vectors at $\bar{P}$ which are orthogonal to the fibre at $\overline{\boldsymbol{P}}$ have components $\left(d x^{i}, d v^{i}\right)$ such that

$$
d v^{i}+\left\{\begin{array}{c}
i \\
j k
\end{array}\right\} v^{j} d x^{k}=0
$$

The last equation is nothing but the defining equation of the parallel displacement, so the $n$-dimensional plane $H^{n}$ in the tangent space of $T(M)^{n}$ at $P$ and orthogonal to the fibre at $\bar{P}$ is nothing but the horizontal plane in the sense of modern theory of connections based on the theory of fibre bundles.

The set of all horizontal planes $H^{n}$ of $T\left(M^{n}\right)$ constitutes an $n$-dimensional distribution $H$ in $T\left(M^{n}\right)$.

THEOREM 1. The distribution $H$ in $T\left(M^{n}\right)$ is involutive if and only if the Riemannian manifold $M^{n}$ is flat.

Proof. By virtue of (3.9) we see that

$$
X_{a}:\left(\delta_{u}^{i},-\left\{\begin{array}{c}
i \\
j a
\end{array}\right\} v^{j}\right) \quad(a, b, c=1, \ldots, n)
$$

are $n$ vector fields of $T\left(M^{n}\right)$ such that they span the $n$-dimensional plane of $H$ at every point of $T\left(M^{n}\right)$. So, to prove Theorem 1 it is sufficient to show that $\left[X_{a}, X_{b}\right]$ is a linear combination of $X_{c}$, i.e. a relation of the form

$$
\left[X_{a}, X_{b}\right]=\alpha_{a b}{ }^{r} X_{c}
$$

holds good if and only if $M$ is flat, where $\left[X_{a}, X_{b}\right]$ means the Poisson bracket of $T\left(M^{n}\right)$.

Now, we can easily verify that

$$
\begin{aligned}
& {\left[X_{a}, X_{b}\right]^{i}=0,} \\
& {\left[X_{a}, X_{b}\right]^{i+i}=K_{\lambda a b}^{i} v^{\prime} .}
\end{aligned}
$$

Hence, if $H$ is involutive, then we may easily see that $R_{\lambda x b}^{t}=0$.

Conversely, if $M^{n}$ is flat, it is clear that $H$ is involutive.

Q.E.

Let $U$ be a coordinate neighborhood of $M^{n}$ with coordinates $x^{i}$ and
C: $x^{i}=x^{i}(t)$
$a \leqq t \leqq b$

be a curve of class $C^{1}$ in $U$. Through any point $\left(x^{i}(0), v^{i}(0)\right)$ of $U \times E^{n}$, there passes a curve

$$
\bar{C}: x^{i}=x^{i}(t), v^{i}=v^{i}(t), a \leqq t \leqq b,
$$

which satisfies the differential equation

$$
\frac{d v^{i}}{d t}+\left\{\begin{array}{l}
i \\
j k
\end{array}\right\} v^{j} \frac{d x^{k}}{d t}=0 .
$$

The curve $\bar{C}$ is characterized geometrically by the fact that the projection 
of the point $\bar{P}(t)$ corresponding to the value $t$ of $\bar{C}$ lies on the fibre passing through the point $P(t)$ of $M^{n}$ corresponding to the same value of $t$ of $C$ and the tangent vector of $\bar{C}$ at $\bar{P}(t)$ lies in the horizontal plane at $\bar{P}(t)$. We shall call such a curve $\bar{C}$ as a lift of $C$. Every lift of a curve $C$ of $M^{n}$ induces a vector field $v^{i}(t)$ along $C$, which we shall call the induced vector field by the lift.

Of course the notion of a lift of $C$ can be naturally extended to any curve $C$ of class $C^{1}$ which is not contained in a single coordinate neighborhood of $M^{n}$. The following theorem is an immediate consequence of our definition.

THEOREM 2. If a curve $\bar{C}$ of $T\left(M^{n}\right)$ is a lift of a curve $C$ in $M^{n}$, the vector field intuced by $C$ is parallel along $C$ in Levi-Civita's sense. Conversely, any vector field parallel along $C$ in Levi-Civita's sense can be regarded as a vetcor field induced by a lift of $C$.

Any curve $\bar{C}$ of $T\left(M^{n}\right)$ such that the tangent vec or at every point $\overline{P(t)}$ of it lies in the horizontal plane at $\bar{P}(t)$, in other words orthogonal to the fibre, is called a horizontal curve.

Theorem 3. Any curve $\bar{C}$ of $T\left(M^{n}\right)$ is horizontai i.e. orthogonal to the fibres it passes, if and only if it is a lift of a curve of $M^{n}$.

4. The geodesic flow. The differential equations of geodesics in $M^{n}$ with respect to a coordinate neighborhood $U\left(x^{i}\right)$ can be written as

$$
\left\{\begin{array}{l}
\frac{d x^{i}}{d t}=v^{i}, \\
\frac{d v^{i}}{d t}=-\left\{\begin{array}{c}
i \\
j k
\end{array}\right\} v^{j} v^{k} .
\end{array}\right.
$$

If we take an open covering $\left\{U_{\lambda}\right\}\left(\lambda \in J, J\right.$ an index set) of $M^{n}$ and consider vector fields on $U_{\lambda} \times E^{n}$ having components

$$
\left(v^{i},-\left\{\begin{array}{c}
i \\
j k
\end{array}\right\} v^{j} v^{k}\right)
$$

for every $U_{\lambda}$ of the covering, then by the Corollary of Lemma 3, we can see that all of these vector fields for every $U_{\lambda} \times E$ are unified to a vector field over $T\left(M^{n}\right)$.

Let $\bar{P}_{0}$ be a point of $T\left(M^{n}\right)$. Then, there exists one and only one solution $\bar{P}(t)$ of (4.1) which has the initial condition "for $t=0, \bar{P}^{i}(0)=\bar{P}_{0}$ ". If we think $\bar{P}_{0}$ as an arbitrary point of $T\left(M^{n}\right)$, then the correspondence $\overline{P_{0}} \rightarrow \bar{P}(t)$ defines a homeomorphism $T_{t}$ of $T\left(M^{n}\right)$ for every value of $t$. The homeomorphisms $T_{t}(-\infty<t<+\infty)$ constitute an Abelian group which we shall call the geodesic flow of $M^{n}$. $\bar{P}(t)(-\infty<t<\infty)$ describes a curve which we shall call a trajectory of the geodesic flow.

As the vector field having components (4.2) in every $U_{\lambda} \times E^{n}$ defines the geodesic flow, we shall call the vector field as GF-vector field for brevity. 
We get easily the following

THEOREM 4. In the tangent bundle $T\left(M^{n}\right)$ of any Riemannian manifold $M^{n}$, every trajectory of the geodesic flow is a lift of a geodesic of $M^{n}$ such that the induced vector field along the geodesic is the tangent vector field of the geodesic. It is orthogonal to the fibre it meets.

The covariant components of the $G F$-vector field is easily seen to be

$$
\left(g_{i j}(x) v^{j}, 0\right) \text {. }
$$

In 1 we have defined the incompressibility of vector fields. Using this notion, we shall prove a theorem which is an analogue of a theorem of Poincaré for the case of a surface in Euclidean space $E^{3}$. We begin with a lemma.

Lemma 5.

$$
\begin{aligned}
& \frac{\partial G_{J K}}{\partial x^{i}} G^{J K}=4\left\{\begin{array}{c}
h \\
h i
\end{array}\right\}, \\
& \partial G_{J K} G^{J K}=0 . \\
& \partial v^{i}
\end{aligned}
$$

Proof. If we put (3.5) and (3.7) into the left hand side of (4.3), we get

$$
\begin{gathered}
\partial\left(g_{j k}+g_{\beta \gamma}\left\{\begin{array}{c}
\beta j \\
\mu x^{i}
\end{array}\right\}\left\{\begin{array}{c}
\gamma \\
\nu k
\end{array}\right\} v^{\mu} v^{\nu}\right) g^{j k}-2 \frac{\partial([\lambda j, h]}{\partial x^{i}} \underline{\left.v^{\lambda}\right)}\left\{\begin{array}{c}
h \\
\nu k
\end{array}\right\} g^{j k} v^{\nu} \\
+\frac{\partial g_{\beta \gamma}}{\partial x^{i}}\left(g^{\beta \gamma}+g^{k l}\left\{\begin{array}{c}
\beta \\
\mu, k
\end{array}\right\}\left\{\begin{array}{c}
\gamma \\
\nu l
\end{array}\right\} v^{\mu} v^{\nu}\right)
\end{gathered}
$$

which can be easily simplified and gives the value $4\left\{\begin{array}{c}\boldsymbol{h} \\ \boldsymbol{h} i\end{array}\right\}$.

In the same way we can prove (4.4), the calculation is simpler than the case (4.3).

THEOREM 5. The geodesic flow is incompressible.

Proof. To prove the theorem it is sufficient to show that the $G F$-vector field in $T\left(M^{n}\right)$ is incompressible. We put

$$
\bar{\xi}^{i}=v^{i}, \quad \bar{\xi}^{n+i}=-\left\{\begin{array}{c}
i \\
j k
\end{array}\right\} v^{j} v^{k} .
$$

Then the incompressibility of $G F$-vector field is expressed as

$$
\bar{\xi}_{, J}^{I} \equiv \frac{\partial \bar{\xi}^{I}}{\partial x^{I}}+\left\{\begin{array}{l}
I \\
I J
\end{array}\right\} \bar{\xi}^{J}=0
$$

The last equation is transformed to

$$
\frac{\partial \bar{\xi}^{I}}{\partial x^{I}}+\frac{1}{2} \frac{\partial G_{J K}}{\partial x^{I}} G^{J K} \bar{\xi}^{I}=0 .
$$

Hence, by Lemma 5 , we see that

$$
\bar{\xi}_{, I}^{I}=\frac{\partial v^{i}}{\partial x^{i}}+\frac{\partial\left(-\left\{\begin{array}{c}
i \\
j k
\end{array}\right\} \boldsymbol{v}^{j} v^{k}\right)}{\partial \bar{v}^{i}}+2\left\{\begin{array}{c}
h \\
h i
\end{array}\right\} v^{i}=0,
$$


which is to be proved.

5. Theorems on vector fields.

LEMMA 6 . In order that the extended vector field $\bar{\xi}$ of a covariant vector field $\xi$ of $M^{n}$ be a gradient, it is necessary and sufficient that $\xi$ itself is a gradient.

Proof. By virtue of the definition (2.7) of $\bar{\xi}$ we can easily see that our assertion is true. $\left(\xi_{i}, 0\right)$.

REMARK. The Lamma is also true if we replace $\bar{\xi}$ by the vector field

THEOREM 6. In order that the exiended vector field $\bar{\xi}$ of a contravariant vector field $\xi$ of $M^{n}$ be an insompressible vector field, it is necessary and sufficient that $\xi$ itself is an incompressible vector field.

Proof. The divergence of a vector field $\bar{\xi}$ of $T\left(M^{n}\right)$ is given by

$$
\bar{\xi}_{, I}^{L}=\frac{\partial \overline{\boldsymbol{\xi}}^{i}}{\partial \boldsymbol{x}^{i}}+\frac{\partial \overline{\boldsymbol{\xi}}^{n+i}}{\partial \overline{\boldsymbol{v}}^{i}}+\frac{1}{2} \frac{\partial \boldsymbol{G}_{J K}}{\partial \boldsymbol{x}^{\underline{T}}} \boldsymbol{G}^{J K \boldsymbol{\xi}^{\prime}} \text {. }
$$

By virtue of $(4,3),(4.4)$ and $(2.6)$, we can transform the last equation easily to

$$
\begin{aligned}
\bar{\xi}_{, I} & =\frac{\partial \bar{\xi}^{i}}{\partial x^{i}}+\frac{\partial \bar{\xi}^{n+i}}{\partial v^{i}}+2\left\{\begin{array}{c}
i \\
i k
\end{array}\right\} \xi^{k} \\
& =2 \xi_{, i}^{i},
\end{aligned}
$$

which proves our assertion.

THEOREM 7. Let $\xi^{i}$ be a vector field over $M^{n}$. Then, the vector field $\left(0, \xi^{i}\right)$ over $T\left(M^{n}\right)$ is always incompressible.

Projf. By virtue of (4.3), (4.4) and (5.1) we can easily see that our assertion is true.

THEOREM 8. Let $M^{n}$ bo a Riemannian manifold whose Ricci tensor is equal to zero. Then, the extented vector field of a covariant harmonic vector field $\xi$ of $M^{n}$ is harmonic in $T\left(M^{n}\right)$ too.

PROOF. The conditions that a covariant vector field $\xi$ of $M^{n}$ and its extended vector field $\bar{\xi}$ of $T\left(M^{n}\right)$ be harmonic are given by

$$
\xi_{i, j}=\xi_{j, i}, \quad \xi_{, i}^{i}=0 \quad\left(\xi^{i}=g^{i j} \xi_{j}\right),
$$

and

$$
\bar{\xi}_{, J}=\bar{\xi}_{J, I}, \quad \hat{\xi}_{, I}^{I}=0 \quad\left(\hat{\xi}^{I}=G^{I J} \bar{\xi}_{J}\right)
$$

respectively. By virtue of Lemma 6, (5.4), follows from (5.3) . Although $\bar{\xi}$ is an extension of a covariant vector field $\xi$ of $M^{n}, \hat{\xi}$ is not an extension of the contravariant vector field $\xi$ because the contravariant fundamental tensor field $G^{I J}$ is not the extended tensor field of the tensor field $g^{i j}$. Hence, (5.4) 
is not a direct consequence of Theorem 6 . We have to prove (5.4) by another way.

As we have seen in (5.2),

$$
\hat{\xi}_{, I}^{I}=\frac{\partial \hat{\xi}^{i}}{\partial x^{i}}+\frac{\partial \hat{\xi}^{n+i}}{\partial \boldsymbol{v}^{i}}+2\left\{\begin{array}{c}
i \\
i k
\end{array}\right\} \hat{\xi}^{k}
$$

where $\hat{\xi}^{i}$ and $\hat{\xi}^{n+i}$ are given by

$$
\begin{aligned}
& \hat{\xi}^{i}=G^{i j} \bar{\xi}_{j}+G^{i n+j \bar{\xi}_{n+j},} \\
& \hat{\xi}^{n+i}=G^{n+i} \bar{j} \bar{\xi}_{j}+G^{n+i n+j} \bar{\xi}_{n+j} .
\end{aligned}
$$

Replacing (2.7) and (3.7) in the right hand side of the last two equations, we see easily that

$$
\left\{\begin{array}{l}
\hat{\xi}_{i}=\xi_{, \lambda}^{i} v^{\lambda}, \\
\hat{\xi}^{n+i}=\xi^{i}-\left\{\begin{array}{c}
i \\
j \lambda
\end{array}\right\} \xi_{, \mu}^{j} v^{\lambda} v^{\mu} .
\end{array}\right.
$$

Putting these in (5.5) and making use of the relation

$$
\xi_{;}^{i} i=\xi_{, \lambda i}^{i}-\xi_{, i \lambda}^{i}=R_{j \lambda i}^{i} \xi^{j},
$$

we get

$$
\hat{\xi}_{, I}^{I}=R_{j k} \xi^{j} v^{k} .
$$

By assumption $R_{j k}=0$, hence we see that

$$
\hat{\xi}_{I}^{I}=0 \text {. }
$$

Q.E. D.

THEOREM 9. Let $\xi_{i}$ be a covariant vector field over $M^{n}$. Then, the vector field $\left(\xi_{i}, 0\right)$ over $T\left(M^{n}\right)$ is harmonic in $T\left(M^{n}\right)$ if and only if $\xi_{i}$ is harmonic in $M^{n}$.

Proof. As $\xi_{i}$ is harmonic in $M^{n}, \xi_{i}$ is a gradient in $M^{n}$. Hence, by the remark after Lemma $6,\left(\xi_{i}, 0\right)$ is a gradient in $T\left(M^{n}\right)$.

Now the contravariant components of $\left(\xi_{i}, 0\right)$ are easily seen to be $\left(\xi^{i},-\left\{\begin{array}{c}i \\ k \lambda\end{array}\right\} g^{j k} v^{\lambda} \xi_{j}\right)$, where we put $\xi^{i}=g^{i j} \xi_{j}$. Accordingly, we see that the divergence of the vector field of $T\left(M^{n}\right)$ in consideration is given by

$$
\begin{aligned}
\frac{\partial \xi^{i}}{\partial x^{i}} & -\left\{\begin{array}{c}
i \\
k i
\end{array}\right\} g^{j k} \xi_{j}+2\left\{\begin{array}{c}
h \\
i h
\end{array}\right\} \xi^{i} \\
& =\frac{\partial \xi^{i}}{\partial x^{i}}+\left\{\begin{array}{c}
h \\
i h
\end{array}\right\} \xi^{i} \\
& =\xi_{, i}^{i}=0 .
\end{aligned}
$$

Hence, $\left(\xi_{i}, 0\right)$ is a harmonic vector field over $T\left(M^{n}\right)$. The proof of the converse is easy.

6. Group of isometries. Let $U$ and $U^{\prime}$ be two coordinate neighborhoods of $M^{n}$ with coordinates $x^{i}$ and $x^{\prime a}$ respectively. It is well known that the mapping $f$ : 


$$
x^{\prime a}=x^{\prime a}\left(x^{1}, \ldots, x^{n}\right) \quad x^{i} \in U
$$

of $U$ onto $U^{\prime}$ is an isometry of $U$ onto $U^{\prime}$ if and only if the relation

$$
g_{a b}^{\prime}\left(x^{\prime}\right)=\frac{\partial x^{i}}{\partial x^{\prime a}}-\frac{\partial x^{j}}{\partial x^{\prime b}} g_{i j}(x)
$$

holds good.

THEOREM 10. In order that the extended transformation $\bar{f}$ :

$$
\left\{\begin{array}{l}
x^{\prime a}=x^{\prime a}(x) \quad x \in U \\
v^{\prime a}=-\frac{\partial x^{\prime a}}{\partial x^{i}} v^{i}
\end{array}\right.
$$

of $f$ is an isometry of $U \times E^{n}$ onto $U^{\prime} \times E^{n}$ of $T\left(M^{n}\right)$, it is necessary and sufficient that $f$ itself is an isometry of $U$ onto $U^{\prime}$.

PROOF. If we put

$$
x^{n+i}=v^{i}, x^{\prime n+a}=v^{\prime a}
$$

and write (6.3) as

$$
x^{\prime A}=x^{\prime A}\left(x^{1}, \ldots, x^{2 n}\right),
$$

then it is sufficient to show that the relation

$$
G_{A B}^{\prime}\left(x^{\prime}\right)=\frac{\partial x^{\prime}}{\partial x^{\prime A}} \frac{\partial x^{J}}{\partial x^{\prime B}} G_{I J}(x)
$$

holds good when and only when (6.2) holds. We shall first prove the: necessity.

The case where $A=n+a, B=n+b$. In this case, it is clear that (6.5) reduces to (6.2) itself.

The case where $A=n+a, B=b$. In this case, we can easuy verify that $(6.5)$ reduces to

(6.6) $\quad[\rho b, a]^{\prime}\left(x^{\prime}\right) v^{\prime \rho}=\frac{\partial x^{i}}{\partial x^{\prime a}}\left(-\frac{\partial x^{j}}{\partial x^{\prime \prime}}[\lambda j, i] v^{\lambda}+\frac{\partial^{2} x^{j}}{\partial x^{\prime b} \partial x^{\prime \rho}} v^{\prime \rho} g_{i j}\right)$.

On the other hand, when (6.2) holds good we can easily derive from (6.2) the following relation:

(6.7) $[b c, a]^{\prime}\left(x^{\prime}\right)=\frac{\partial x^{i}}{\partial x^{\prime a}}\left(\frac{\partial x^{j}}{\partial x^{j}}-\frac{\partial x^{k}}{\partial x^{\prime c}}[j k, i]+\frac{\partial^{2} x^{j}}{\partial x^{\prime b} \partial x^{\prime c}} g_{i j}\right)$.

If we mutiply $v^{\prime c}$ to both sides of the last equation we get (6.6).

We shall remark here that we may get

$$
\left\{\begin{array}{c}
a \\
b c
\end{array}\right\}^{\prime}\left(x^{\prime}\right)=\frac{\partial x^{\prime a}}{\partial x^{i}}\left(\frac{\partial x^{j}}{\partial x^{\prime b}} \frac{\partial x^{k}}{\partial x^{\prime c}}\left\{\begin{array}{c}
i \\
j k
\end{array}\right\}+\frac{\partial^{2} x^{i}}{\partial x^{\prime b} \partial x^{\prime c}}\right)
$$

by virtue of (6.2) and (6.7).

The case where $A=a, B=b$. In this case, we can easily verify that (6.5) reduces to

$$
\begin{aligned}
& g_{a b}^{\prime}\left(x^{\prime}\right)+g_{\alpha \beta}\left(x^{\prime}\right)\left\{\begin{array}{c}
\alpha \\
\rho a
\end{array}\right\}^{\prime}\left\{\begin{array}{c}
\beta \\
\sigma b
\end{array}\right\}^{\prime} v^{\prime \rho} v^{\sigma} \\
& =\frac{\partial x^{i}}{\partial x^{\prime a}} \frac{\partial x^{j}}{\partial x^{\prime b}}\left(g_{i j}+g_{p q}\left\{\begin{array}{c}
p \\
\lambda i
\end{array}\right\}\left\{\begin{array}{c}
q \\
\mu j
\end{array}\right\} v^{\lambda} v^{\mu}\right)
\end{aligned}
$$




$$
\begin{aligned}
& +\frac{\partial x^{i}}{\partial x^{\prime a}} \frac{\partial^{2} x^{j}}{\partial x^{\prime b} \partial x^{\prime c}} v^{\prime c}[\lambda i, j] v^{\lambda} \\
& +\frac{\partial^{2} x^{i}}{\partial x^{\prime a}} \frac{\partial x^{\prime c}}{\partial c} v^{\prime c} \frac{\partial x^{j}}{\partial x^{\prime b}}[\lambda j, i] v^{\lambda} \\
& +\frac{\partial^{2} x^{i}}{\partial x^{\bar{a} \partial} x^{\prime c}} v^{\prime c} \frac{\partial^{2} x^{j}}{\partial x^{b} \partial x^{\prime l l}} v^{\prime c} g_{i j} \text {. }
\end{aligned}
$$

However, the first term of the left hand side and the first term in the bracket of the right hand side cancel to each other by (6.2). If we put (6.8) into the second term of the left hand side, then we can easily verify that the last equation reduces to an identity. Consequently, (6.5) follows from (6.2), which is to be proved.

The proof of the sufficiency is clear.

The following corollaries are immediate consequences ot Theorem 10.

COROLLARY 1. Suppose $f$ is an isometry of $M^{n}$, then the extended mapping $\bar{f}$ of $f$ is an isometry of $T\left(M^{n}\right)$.

COROLlARY 2. If a Riemannian manifold $M^{n}$ admits an r-parameter Lie group of isometries, then the tangent bundle $T\left(M^{n}\right)$ admits an r-parameter group of isometries too.

Suppose that

$$
x^{i}=x^{i}+\xi^{i}(x) \delta t
$$

be an infinitesimal isometry of $M^{n}$, i. e. $\xi^{i}(x)$ be a Killing vector field of $M^{n}$. Then, the equation corresponding to $(6.3)_{2}$ is easily seen to be

$$
v^{i}=v^{i}+\frac{\partial \xi^{i}}{\partial x^{j}} v^{j} \delta t
$$

So, the equation corresponding to $(6.4)$ is

$$
x^{\prime}=x^{I}+\bar{\xi}^{\prime} \delta t
$$

where $\bar{\xi}^{l}$ is the extension of $\xi^{i}$. Hence, we get the following Corollary.

COROLlaRY 3. In order that an extension $\xi$ of a contravariant vector field $\xi$ of $M^{n}$ be a Killing vector field of $T\left(M^{n}\right)$, it is necessary and svfficient that $\xi$ itself is a Killing vector field of $M^{n}$.

THEOREM 11. If a Riemannian manifold $M^{n}$ admits $a$ parallel vector field $\xi^{i}$, then the tangent bundle $T\left(M^{n}\right)$ admits a Killing vector field $\left(0, \xi^{i}\right)$.

Proof. Consider first a vector field of the type $\left(0, \xi^{n+i}\right)$ and seek for the conditions in order that it is a Killing vector field of $T\left(M^{n}\right)$. In this case the Killing equation of $T\left(M^{n}\right)$ is, analogously to $(1.2)^{\prime \prime}$, given by

$$
\frac{\partial G_{J K}}{\partial v^{i}} \xi^{n+i}+\frac{\partial \xi^{n+i}}{\partial x^{K}} G_{J_{n+i}}+\frac{\partial \xi^{n+i}}{\partial x^{J}} G_{n+i K}=0 .
$$

When $J=j$ and $K=k,(6.10)$ reduces, by virtue of (3.5), to 


$$
\begin{aligned}
& {\left[g_{b c}\left\{\begin{array}{c}
b \\
i j
\end{array}\right\}\left\{\begin{array}{c}
c \\
\nu k
\end{array}\right\} v^{\nu}+g_{b c}\left\{\begin{array}{c}
b \\
\mu j
\end{array}\right\}\left\{\begin{array}{c}
c \\
i k
\end{array}\right\} v^{\mu}\right] \xi^{n+i} } \\
&+\frac{\partial \xi^{n+i}}{\partial x^{k}}[\lambda j, i] y^{\lambda}+\underset{\partial x^{j}}{\partial \xi^{n+i}}[\lambda k, i] v^{\lambda}=0 .
\end{aligned}
$$

The last equation can be easily transformed to

$$
[\lambda j, i] \xi_{, k}^{n+i}+[\lambda k, i] \xi_{, j}^{n+i}=0,
$$

where we have put

$$
\xi^{n+i},{ }_{k}=\frac{\partial \boldsymbol{\xi}^{n+i}}{\partial \boldsymbol{x}^{k}}+\left\{\begin{array}{c}
i \\
j k
\end{array}\right\} \xi^{n+j} .
$$

When $J=j$ and $K=n+k$, (6.10) reduces to

$$
[i j, k] \xi^{n+i}+\frac{\partial \xi^{n+i}}{\partial x^{j}} g_{k i}+\frac{\partial \xi^{n+i}}{\partial v^{k}}[\lambda j, i] v^{\lambda}=0,
$$

which can be transformed to

$$
a_{k i} \xi^{n+i}{ }_{j}+\frac{\partial \xi^{n+i}}{\partial v^{k}}[\lambda j, i] v^{\lambda}=0 .
$$

When $J=\boldsymbol{n}+j, K=\boldsymbol{n}+k,(6.10)$ reduces to

$$
\frac{\partial \xi^{n+i}}{\partial v^{k}} g_{j i}+\frac{\partial \xi^{n+i}}{\partial v^{j}} g_{k i}=0 .
$$

The three equations (6.11) are satisfied if $\xi^{n+i}$ is independent upon $v$ and $\xi^{n+i}, k=0$. Hence, if we replace $\xi^{n+i}$ by a parallel vector field $\xi^{i}$ of $M^{n}$, we see that $\left(0, \xi^{i}\right)$ is a Killing vector field of $T\left(M^{n}\right)$.

Q.E.D.

Theorem 12. Suppose that a Riemannian manifold $M^{n}$ admits $p$ linearly independent parallel vector field $\xi_{a}^{i}(a=1, \ldots, p)$ and $g_{p}$ be the group of translations of $M^{n}$ generated by $X_{a} f=\xi_{a}^{i} \frac{\partial f}{\partial x^{i}}$. If $g_{p}$ is an invariant subgroup of the maximal connected group of isometries $G_{r}$ of $M^{n}$, then the tangent bundle $T\left(M^{n}\right)$ admits an $(r+p)$-parameter group of isometries.

Proof. Let

$$
X_{\lambda}=\xi_{\lambda}^{i} \frac{\partial}{\partial x^{i}}, \quad \lambda, \mu=1,2, \ldots, r
$$

be differential operators which generate $G$. and the first $p$ of them generate $g_{p}$. Of course, $\xi_{\wedge}^{i}$, s are Killing vector fields of $M^{n}$, especially the first $p$ of them are parallel vector fields of $M^{n}$. Then we have a relation of the form

$$
\left[X_{\lambda}, X_{\mu}\right]=c_{\lambda \mu}^{\nu} X_{r},
$$

where $c_{\lambda \mu}{ }^{\nu}$ 's are constants of structure of $G_{r}$.

Now, we put

$$
\begin{aligned}
& \overline{\boldsymbol{X}}_{\boldsymbol{\lambda}}=\xi_{\lambda}^{i} \frac{\partial}{\partial x^{i}}+\left(\frac{\partial \xi_{\lambda}^{i}}{\partial x^{j}} v^{j}\right) \frac{\partial}{\partial \boldsymbol{v}^{i}}, \\
& \bar{Y}_{\boldsymbol{a}}= \\
& \xi_{\imath}^{i} \frac{\partial}{\partial \boldsymbol{v}^{i}},
\end{aligned}
$$


then, by virtue of Corollary 3 of Theorem 10 and Theorem 11, $\overline{X_{\lambda}}$ and $\overline{Y_{a}}$ are $r+p$ linearly independent Killing vector fields of $T\left(M^{n}\right)$.

We want to show that these $r+p$ vector fields generate an $(r+p)$-parameter group.

First, as $\bar{X}_{\lambda}$ 's are extensions of $\boldsymbol{X}_{\lambda}$, it is evident that

$$
\left[\bar{X}_{\lambda}, \bar{X}_{\mu}\right]=c_{\lambda \mu}{ }^{\nu} \bar{X}_{\nu}
$$

In the next place, we can show that

$$
\begin{aligned}
& {\left[\bar{X}_{\lambda}, \bar{Y}_{b}\right]^{i}=0,} \\
& {\left[\bar{X}_{\lambda}, \bar{Y}_{b}\right]^{n+i}=c_{\lambda b}{ }^{c} \xi_{c}^{i},}
\end{aligned}
$$

by virtue of the assumption that $g_{p}$ is an invariant subgroup of $G_{r}$. Hence we get

$$
\left[\bar{X}_{\lambda}, \bar{Y}_{b}\right]=c_{\lambda b} c \bar{Y}_{c}
$$

Finally we can easily see that

$$
\left[\bar{Y}_{a}, \bar{Y}_{b}\right]=0 .
$$

The three equations (6.12) show that $\bar{X}_{\lambda}, \bar{Y}_{a}$ generate an $(r+p)$-parameter group of isometries of $T\left(M^{n}\right)$.

Q.E.D.

All isometries of $T\left(M^{n}\right)$ treated in this section are fibre preserving isometries. It may be a problem to study isometries of $T\left(M^{n}\right)$ which are not fibre preserving.

7. Geodesics of $\boldsymbol{T}\left(\boldsymbol{M}^{n}\right)$. First we shall calculate Christoffel's symbols of the fundamental metric tensor $G_{J K}$ of $T\left(M^{n}\right)$. By virtue of (3.5) and

$$
\overline{[J K, H]}=\frac{1}{2}\left(\frac{\partial G_{J H}}{\partial x^{K}}+\frac{\partial G_{H K}}{\partial x^{\prime}}-\frac{\partial G_{J K}}{\partial x^{H}}\right),
$$

we can verify that

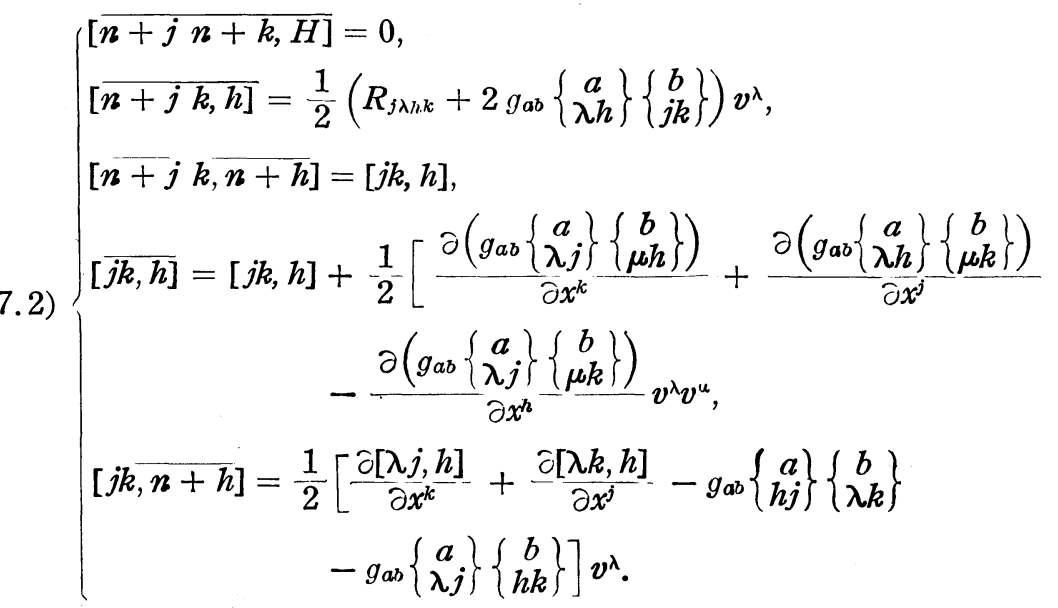

By virtue of (7.2) and 


$$
\overline{\left\{\begin{array}{l}
I \\
J K
\end{array}\right\}}=G^{I \theta}[J \overline{K, H]},
$$

we can verify, after some long calculations, that

$$
\begin{aligned}
& \left\{\begin{array}{l}
\left\{\begin{array}{l}
I n+j n+k \\
\{n
\end{array}=0,\right. \\
\overline{\left\{\begin{array}{c}
i \\
n+j k
\end{array}\right\}}=\frac{1}{2} R_{k j \lambda}^{i} v^{\lambda}, \\
\left\{\begin{array}{c}
n+i \\
n+j k
\end{array}\right\}=\left\{\begin{array}{c}
i \\
j k
\end{array}\right\}-\frac{1}{2}\left\{\begin{array}{c}
i \\
\mu h
\end{array}\right\} R_{k j, \lambda}^{h} v^{\lambda} v^{\mu},
\end{array}\right. \\
& \overline{\left\{\begin{array}{c}
i \\
j k
\end{array}\right\}}=\left\{\begin{array}{c}
i \\
j k
\end{array}\right\}+\frac{1}{2}\left(R_{k h_{\mu}}^{i}\left\{\begin{array}{c}
h \\
\lambda j
\end{array}\right\}+R_{j h \mu}^{i}\left\{\begin{array}{c}
h \\
\lambda k f
\end{array}\right) v^{\lambda} v^{\mu},\right. \\
& \overline{\left\{\begin{array}{c}
n+i \\
j k
\end{array}\right\}}=\frac{1}{2}\left(R_{j \lambda k}^{i}+R_{k \lambda j}^{i}+2 \frac{\partial\left\{\begin{array}{c}
i \\
j k
\end{array}\right\}}{\partial x^{\prime}}\right) v^{\lambda} \\
& +\frac{1}{2}\left\{\begin{array}{c}
i \\
\nu h
\end{array}\right\}\left(R_{k \mu l}^{h}\left\{\begin{array}{c}
l \\
\lambda j
\end{array}\right\}+R_{j \mu l}^{h}\left\{\begin{array}{c}
l \\
\lambda k
\end{array}\right\}\right) v^{\lambda} v^{u} v^{\nu} .
\end{aligned}
$$

Now, we shall express the differential equation of geodesics of $T\left(M^{n}\right)$, i.e.

$$
\begin{gathered}
d^{2} x^{T} \\
d \sigma^{2}
\end{gathered}+\left\{\begin{array}{c}
I \\
J K
\end{array}\right\} \frac{d x^{J}}{d \sigma}-\frac{d x^{K}}{d \sigma}=0
$$

in terms of quantities of $M^{n}$.

When $I=i,(7.5)$ can be rewritten, by virtue of (7.4), as

$$
\begin{aligned}
\frac{d^{2} x^{i}}{d \sigma^{2}}+\left[\left\{\begin{array}{c}
i \\
j k
\end{array}\right\}\right. & \left.+\frac{1}{2}\left(R_{k: x \mu}^{i}\left\{\begin{array}{c}
a \\
\lambda j
\end{array}\right\}+R_{j a \mu}^{i}\left\{\begin{array}{c}
a \\
\lambda k
\end{array}\right\}\right) v^{\lambda} v^{\mu}\right] \frac{d x^{j}}{d \sigma} \frac{d x^{k}}{d \sigma} \\
& +R_{j k \lambda}^{i} v^{\lambda} \frac{d x^{j}}{d \sigma} \frac{d x^{k}}{d \sigma}=0 .
\end{aligned}
$$

This is easily transformed to

$$
\frac{d^{2} x^{i}}{d \sigma^{2}}+\left\{\begin{array}{c}
i \\
j k
\end{array}\right\} \frac{d x^{j}}{d \sigma} \frac{d x^{k}}{d \sigma}=R_{j \lambda \mu}^{i} \frac{d x^{j}}{d \sigma} v^{\lambda} \frac{D v^{\mu}}{d \sigma} .
$$

When $I=n+i,(7.5)$ can be rewritten, by virtue of (7.4), as

$$
\begin{aligned}
\frac{d^{2} v^{i}}{d \sigma^{2}} & +\frac{1}{2}\left[\left(R_{j \lambda k}^{i}+R_{k \lambda j}^{i}+2 \frac{\partial\left\{\begin{array}{c}
i \\
j k
\end{array}\right\}}{\partial x^{\lambda}}\right) v^{\lambda}\right. \\
& \left.+\left\{\begin{array}{c}
i \\
\nu l
\end{array}\right\}\left(R_{k \mu \lambda}^{\prime}\left\{\begin{array}{c}
a \\
\lambda j
\end{array}\right\}+R_{j \mu \lambda}^{l}\left\{\begin{array}{c}
a \\
\lambda k
\end{array}\right\}\right) v^{i} v^{\mu} v^{\nu}\right] \frac{d x^{j}}{d \sigma} \frac{d x^{k}}{d \sigma} \\
& +2\left[\left\{\begin{array}{c}
i \\
j k
\end{array}\right\}-\frac{1}{2}\left\{\begin{array}{c}
i \\
\mu h h
\end{array}\right\} R_{j k \lambda}^{h} v^{\prime} v^{\mu}\right] \frac{d x^{j}}{d \sigma} \frac{d x^{k}}{d \sigma}=0 .
\end{aligned}
$$

However, if we use the relations

$$
\begin{aligned}
& \frac{D v^{i}}{d \sigma}=\frac{d v^{i}}{d \sigma}+\left\{\begin{array}{c}
i \\
j k
\end{array}\right\} v^{j} \frac{d x^{k}}{d \sigma}, \\
& \frac{D^{2} v^{i}}{d \sigma^{2}}=\frac{d^{2} v^{i}}{d \sigma^{2}}+\frac{d}{d \sigma}\left(\left\{\begin{array}{c}
i \\
j k
\end{array}\right\} v^{j} \frac{d x^{k}}{d \sigma}\right)+\left\{\begin{array}{c}
i \\
l m
\end{array}\right\}-\frac{D v^{l}}{d \sigma} \frac{d x^{m}}{d \sigma}
\end{aligned}
$$


and eliminate $\frac{d^{2} v^{i}}{d \sigma^{2}}$ from (7.6), we see that (7.6) reduces to a simple form

$$
\frac{D^{2} v^{i}}{d \sigma^{2}}=0 \text {. }
$$

Hence, the differential equation of geodesics of $T\left(M^{n}\right)$ becomes as follows.

$$
\left\{\begin{array}{c}
\frac{d^{2} x^{i}}{d \sigma^{2}}+\left\{\begin{array}{c}
i \\
j k
\end{array}\right\} \frac{d x^{j}}{d \sigma} \frac{d x^{k}}{d \sigma}=R_{j \lambda \mu}^{i} \frac{d x^{j}}{d \sigma} v^{\lambda} \frac{D v^{\mu}}{d \sigma}, \\
\frac{D^{2} v^{i}}{d \sigma^{2}}=0 .
\end{array}\right.
$$

THEOREM 13. Every lift of a geodesic of a Riemannian manifold $M^{n}$ is a geodesic of the tangent bundle $T\left(M^{n}\right)$.

PROOF. For the lift in consideration, the relations

$$
\begin{gathered}
\frac{d^{2} x^{i}}{d \sigma^{2}}+\left\{\begin{array}{c}
i \\
j k
\end{array}\right\} \frac{d x^{j}}{d \sigma} \frac{d x^{k}}{d \sigma}=0, \\
\frac{D v^{i}}{d \sigma}=0
\end{gathered}
$$

hold good. Hence, (7.7) is satisfied identically.

Q.E.D.

By virtue of Theorem 4 we get the following

COROLlarY. Every trajectory of the geodesic flow of a Riemannian manifold $M^{n}$ is a geodesic of the tangent bundle $T\left(M^{n}\right)$.

THEOREM 14. The necessary and sufficient condition that a curve $C$ of $M^{n}$ has a lift which is a geodesic of $T\left(M^{n}\right)$ is that $C$ itself is a geodesic of $M^{n}$.

The proof is evident.

We shall call a curve in $M^{n}$ which may be regarded as the image of a geodesic of $T\left(M^{n}\right)$ as a submarine geodesic of $M^{n}$. Of course, every geodesic of $M^{n}$ is a submarine geodesic of $M^{n}$.

THEOREM 15. If $M^{n}$ is a flat Riemannian manifold, then every submarine geolesic is a geodesic of $M^{n}$.

The proof is evident.

A curve in $M^{n}$ is said to be a spacial curve if the development of the curve in the tangent space of a point on the curve lies in a three dimensional linear subspace of the tangent space. Then, we get the following

THEOREM 16. If $M^{n}$ is a Riemannian manifold of constant curvature $K$ $(K \neq 0)$, then every submarine geodesic is a spacial curve.

Proof. As $M^{n}$ is of constant curvature,

$$
R_{j k l}^{i}=K\left(\delta_{k}^{i} g_{j l}-\delta_{i}^{i} g_{j k}\right)
$$

Hence, (7.7) reduces to 


$$
\left\{\begin{array}{c}
\frac{d^{2} x^{i}}{d \sigma^{2}}+\left\{\begin{array}{c}
i \\
j k
\end{array}\right\} \frac{d x^{j}}{d \sigma} \frac{d x^{k}}{d \sigma}=\left(K g_{j k} \frac{d x^{j}}{d \sigma} \frac{D v^{k}}{d \sigma}\right) v^{i}-\left(K g_{j k} \frac{d x^{j}}{d \sigma} v^{k}\right) \frac{D v^{i}}{d \sigma} \\
\frac{D^{2} v^{i}}{d \sigma^{2}}=0
\end{array}\right.
$$

The last equation shows that covariant derivatives of higher orders of $\frac{d x^{i}}{d \sigma}$ are always linear combinations of $v^{i}$ and $\frac{D v^{i}}{d \sigma}$. Hence, the $\pi$-image of any geodesic of $T\left(M^{n}\right)$ is a spacial curve.

Now, as every fibre of the bundle $T\left(M^{n}\right)$ is a tangent space of $M^{n}$ we may speak of straight lines on a fibre.

THEOREM 17. Every straight line on a fibre of the tangent bundle $T\left(M^{n}\right)$ is a geodesic of $T\left(M^{n}\right)$.

PROof. Let $U$ be a coordinate neighborhood of $M^{n}$ containing the point $x_{0}^{i}$ which is the projection of the fibre. Then the straight line in consideration can be expressed as

$$
x^{i} \equiv x_{0}^{i}, \quad v^{i}=a^{i} t+b^{i}
$$

where $x_{0}^{i}, a^{i}$ and $b^{i}$ are constants. Hence, (7.7) is satisfied identically.

THEOREM 18. Every fibre of the tangent bundle $T\left(M^{n}\right)$ of a Riemannian manifold $\mathrm{M}^{n}$ is a totally geodesic submanifold of $T\left(M^{n}\right)$.

PRoof. The induced metric on every fibre is easily seen to be Euclidean. So any straight line is a geodesic of the fibre with the induced metric from $T\left(M^{n}\right)$. Hence, by virtue of Theorem 17, every geodesic of the fibre with the induced metric is also a geodesic of $T\left(M^{n}\right)$, therefore every fibre is a totally geodesic submanifold of $T\left(M^{n}\right)$.

In this paper we have studied only properties concerning the metric tensor and Christoffel's symbols of $T\left(M^{*}\right)$. We shall leave the discussion of properties on the curvature tensor in later papers.

MATHEMATICAL INSTITUTE, TôHOKU UNIVERSITY. 